\title{
Nondestructive inspection of port operation machinery based on Modified Multifractal Algorithm
}

\author{
GuoXu $^{1, a}$, LiuLeiLei ${ }^{2, b}$ and SunLei, ${ }^{3, c}$
}

${ }^{1,2,3}$ Key Laboratory of Environmental Protection in Water Transport Engineering Ministry of Communications, Tianjin Research Institute of Water Transport Engineering, Tianjin 300456, China.

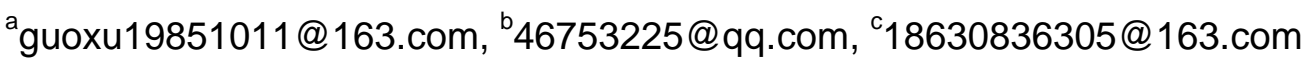

Keywords: multifractal;nondestructive inspection

Abstract. This Nondestructive inspection of mechanical equipment is to diagnose the mechanical the wear and corrosion of the machine under the condition of no disassembly. With the rapid development of port industry, the number of all kinds of port operating machinery is increasing, and the complexity of the equipment is also increasing. In this paper, a new method for nondestructive inspection of port equipment is proposed by improving the existing multifractal algorithm.

\section{Differential Box Counting method}

DBC(Differential Box Counting method) $)^{[1-2]}$ is first proposed by N.Sarkar and B. Chaudhuri ${ }^{[3-4]}$, Based on the self-similarity of fractal. The gray image is imagined as a three-dimensional image filled with several boxes, in which the image height is the gray value of each pixel.

The Basic principle of DBC is as follows:firstly, the two-dimensional gray scale of $M \times M$ pixels is divided into grids of $s \times s$ pixels. Two dimensional gray image of $M \times M$ pixels is considered as a surface in 3D space,the length and width of the image represent the $\mathrm{X}$ and $\mathrm{Y}$ axes of the three-dimensional coordinate system respectively, and the gray value of each pixel in the image represents the $\mathrm{Z}$ axis. Boxes(hight h) are stacked on the grids of $s \times s$ pixels. Calculation method of boxes are as follows:

$$
h=\frac{s}{M} \cdot G
$$

Where:

$\mathrm{s}=$ The width of a box $(s \times s$ grid $)$

$\mathrm{M}=$ width of image $(M \times M$ image $), 2 \leq s \leq M / 2$

$\mathrm{G}=$ gray value of image.

Set the maximum and minimum values of gray values in the $(\mathrm{x}, \mathrm{y})$ grid in the $k$ and $l$ boxes.

$$
\begin{gathered}
\mathrm{n}_{\mathrm{s}}(\mathrm{i}, \mathrm{j})=\mathrm{k}-\mathrm{l}+1 \\
N_{s}=\sum n_{s}(i, j)
\end{gathered}
$$

Where

$n_{s}(i, j)=$ Number of boxes covered in the $(i, j)$ grid, Number of boxes in the entire image.

$\mathrm{N}_{s} \quad=$ Number of boxes in the entire image.

Using different $s$, the results of fractal dimension is calculated by least squares fitting are as follows:

$$
D=\lim _{s \rightarrow 0} \frac{\log \left(N_{s}\right)}{\log (1 / s)}
$$




\section{Multiracial Differential Box Counting method}

The DBC only describes the geometric structure of the object, but it can not describe the fractal dimension of the image from multi angle, so the $\mathrm{MDBC}^{[3-4]}$ (Multiracial Differential Box Counting method) must be introduced.

$\mathrm{MDBC}$ is similar to $\mathrm{DBC}$, it also needs to establish a three-dimensional space, set the box in the grid is $B_{i}$, the number of pixels in the image of the boxes is $u\left(B_{i}\right)$, the gray value of a certain scale $\mathrm{A}$ is as follows:

$$
u(A)=\sum u\left(B_{i}\right)
$$

Set

$$
p(i)=\frac{u\left(B_{i}\right)}{u(A)}
$$

Then the multifractal dimension is as follows:

$$
\begin{cases}D_{q}=\frac{1}{q-1} \lim _{s \rightarrow 0} \frac{\log \sum_{i}^{N_{s}} p(i)_{i}^{q}}{\log s} & \\ D_{1}=\lim _{s \rightarrow 0} \frac{\sum_{i}^{N_{r}} p(i) \log p(i)}{\log s} & q \neq 1\end{cases}
$$




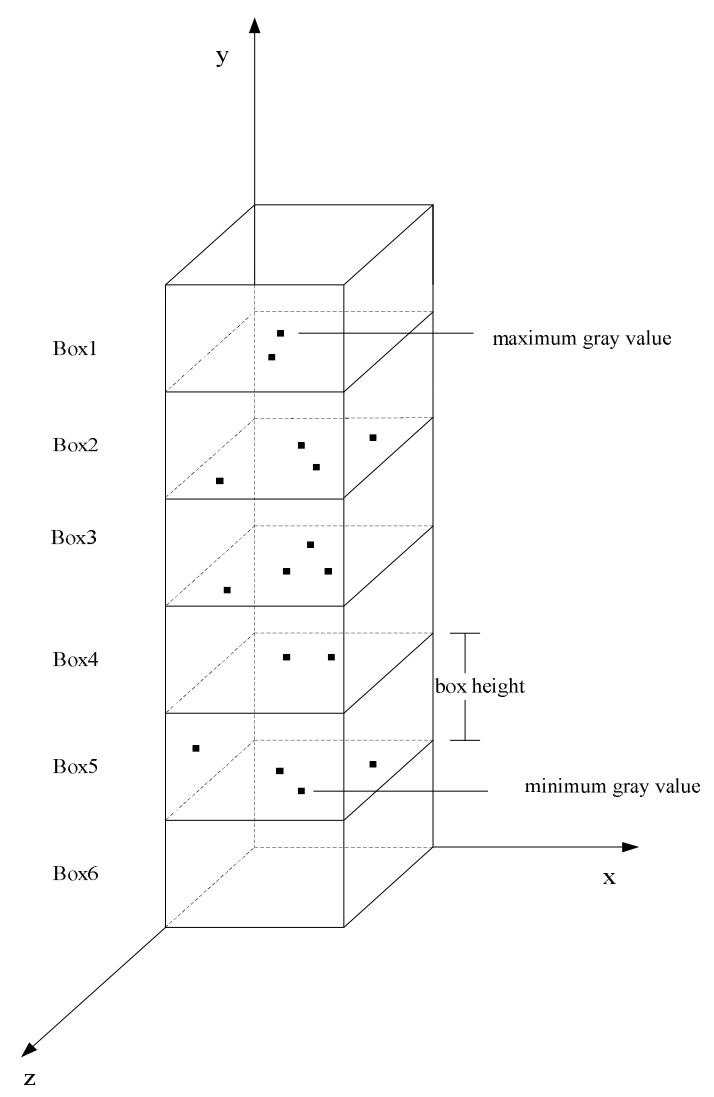

Figure 1 Schematic diagram of algorithm model

\section{Research status at home and abroad}

Kaewaramsri et $\mathrm{a}^{[5]}$ proposes a more accurate FD estimation, an improved triangle box-counting method, to increase the precision of box-counts associated with box sizes. The triangle-box-partition technique provides the double precision for box-counts, thus it can solve the overcounting issue and enhance the accuracy of the FD estimation. The proposed method is evaluated its performance in terms of fitting error. The experimental results show that the proposed method outperforms the existing methods, including differential box-counting (DBC), improved DBC (IDBC), and box-counting with adaptable box height (ADBC) methods.

Shibao Lua et a ${ }^{[6]}$ proposed a method of diagnostic the diagnosis and identification are carried out in combination of the multi-fractal spectrum to provide a new method for fault diagnosis of the hydraulic turbine unit. After the application test, the results show that the method can improve the intelligence and humanization of diagnosis, enhance the man-machine interaction, and produce satisfactory identification result.

H.F. Huang et a ${ }^{[7]}$ proposed a wavelet fractal method, which is proposed by combining the fractal theory with a wavelet decomposition. The proposed method along with the correlation dimension acquired from the generic programming method is applied to characterize the experimental signals under three different conditions. Both methods show the obvious advantages in extracting characteristics from the signals under different conditions. By comparing the statistical data of such two methods, it can be concluded that the characteristics obtained from the wavelet fractal method have shown a high accuracy and thus this method provides a powerful strategy in the fault diagnosis for the practical applications. 
Hongmei Liu et a ${ }^{[8]}$ proposes a method for gearbox fault feature extraction based on empirical mode decomposition (EMD) and multi-fractal detrended cross-correlation analysis (MFDCCA).

\section{Multiracial Differential Box Counting method Based on Entropy (MDBCBE)}

\section{Introduction to algorithm principle}

One of the main problems of Multifractal Algorithm is that the selection of box height is Unreasonable. According to the changing trend of the gray level of the image, the accuracy of multifractal dimension can be improved by adjusting the box height.

Through the the entropy of texture of the image, the gray information of the image, the gray value of each pixel and the gray scale distribution in the neighborhood of the pixel can be obtained. The ratio of the entropy of the texture and the image gray-mean can be used to express the intensity of the gray value of the image. According to the above data to adjust the height of the box, can effectively reduce the "empty box".

This paper chooses the gray value of each pixel in the image and mean of its neighborhood gray value to form two tuple, and the entropy of texture is as follows:

$$
\begin{gathered}
P_{i, j}=\frac{n(i, j)}{M^{2}} \\
H=-\sum_{i} \sum_{j} P_{i, j} I n P_{i, j}
\end{gathered}
$$

Where:

$i=$ gray value of each pixel.

$\mathrm{j}=$ mean value of neighborhood gray

$P_{i, j}=$ the joint probability density of I and $\mathrm{j}$

$n_{i, j}=$ the frequency of $(i, j)$

$H=$ the entropy of texture

Thus, the calculation method of box height in the improved algorithm is as follows:

$$
\begin{gathered}
h=\frac{S}{M} \cdot\left(G_{\max }-G_{\min }\right) \cdot H / \mu \\
\mu=\frac{\sum g}{M^{2}}
\end{gathered}
$$


Where:

$G_{\max }=$ Maximum gray value.

$G_{\min }=$ Minimum gray value

$H=$ the entropy of texture

$\mu=$ gray mean of the image

$g=$ the gray value of each pixel

$M=$ edge of image

Change $n_{s}(i, j)=\frac{\sum g}{M^{2}}$

$n_{s}(i, j)=k \cdot 1+1$ (formula 2) to

$$
n_{s}(i, j)=\operatorname{ceil}\left(\frac{k^{\prime}-l}{h}\right)+1
$$

Where

Ceil $=$ The largest integer less than the number of parentheses.

$k^{\prime}=$ Maximum gray value of pixels in the box of this column

$l^{\prime}=$ Minimum gray value of pixels in the box of this column 


\section{Algorithm comparison and result analysis}

The paper choses 3 images of Brodatz texture ,the result is as follows:

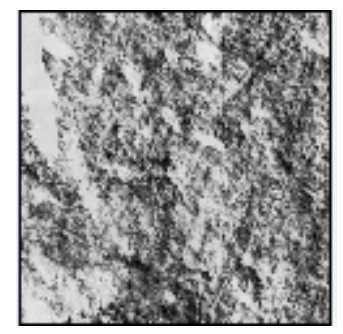

(a) D002

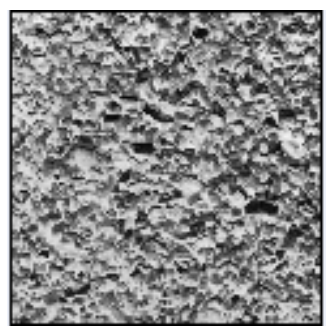

(b) D005

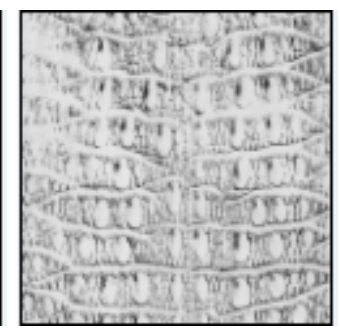

(c) D010

Figure 2 image of Brodatz texture library

Table 1 Comparison of fitting error between MDBCBE algorithm and MDBC algorithm

\begin{tabular}{cccccc}
\hline NO. & $\mathrm{q}$ & \multicolumn{2}{c}{ MDBC } & \multicolumn{2}{c}{ DBMCBE } \\
& & $\mathrm{D}$ & $\mathrm{FE}$ & $\mathrm{D}$ & $\mathrm{FE}$ \\
\hline \multirow{3}{*}{$\mathrm{D} 002$} & 1 & 2.34510 & 0.0697744 & 2.33926 & 0.0500195 \\
& 2 & 2.32479 & 0.0551763 & 2.29957 & 0.0429492 \\
& 3 & 2.29175 & 0.0260702 & 2.25867 & 0.0215090 \\
\hline \multirow{3}{*}{$\mathrm{D} 005$} & 1 & 2.33396 & 0.0778229 & 2.33388 & 0.0539981 \\
& 2 & 2.31252 & 0.0705984 & 2.29420 & 0.0492774 \\
& 3 & 2.28727 & 0.0367526 & 2.25830 & 0.0264158 \\
\hline & 1 & 2.38375 & 0.0601113 & 2.32389 & 0.0234709 \\
$\mathrm{D} 010$ & 2 & 2.38535 & 0.0409577 & 2.26843 & 0.0308709 \\
& 3 & 2.35586 & 0.0173439 & 2.22100 & 0.0147527 \\
\hline
\end{tabular}

Table 2 Time comparison between MDBCBE algorithm and MDBC algorithm

\begin{tabular}{|c|c|c|c|c|c|}
\hline \multirow[t]{2}{*}{ NO. } & \multirow[t]{2}{*}{ q } & \multirow{2}{*}{$\begin{array}{c}\text { MDBC } \\
\text { D }\end{array}$} & \multicolumn{3}{|c|}{ DBMCBE } \\
\hline & & & $\mathrm{T}$ & D & $\mathrm{T}$ \\
\hline \multirow{3}{*}{ D002 } & 1 & 2.3451 & 1531 & 2.33926 & 2162 \\
\hline & 2 & 2.32479 & 1468 & 2.29957 & 1594 \\
\hline & 3 & 2.29175 & 1500 & 2.25867 & 1578 \\
\hline \multirow{3}{*}{ D005 } & 1 & 2.33396 & 1563 & 2.33388 & 2147 \\
\hline & 2 & 2.31252 & 1547 & 2.29420 & 1609 \\
\hline & 3 & 2.28727 & 1531 & 2.2583 & 1563 \\
\hline \multirow{3}{*}{ D010 } & 1 & 2.38375 & 1687 & 2.32389 & 2163 \\
\hline & 2 & 2.38535 & 1563 & 2.26843 & 1609 \\
\hline & 3 & 2.35586 & 1641 & 2.22100 & 1547 \\
\hline
\end{tabular}

Due to the complexity of the algorithm, the MDBCBE algorithm takes a long time, but it can still meet the real-time requirements.

\section{Application of improved algorithm}

Condition monitoring of port machinery and equipment based on Modified Multifractal Algorithm

By using the Modified Multifractal Algorithm, we can obtain the nonlinear signals generated during the operation of port machinery,and compute the multifractal dimension.the equipment can 
be monitored by using the multi fractal dimension of the vibration signal of the machinery, and the failure mode of the equipment.can be determined

\section{The failure of machine wear analysis base on Modified Multifractal Algorithm}

The wear state of machine is obtained by fractal feature of wear debris projection. The fractal dimension is calculated by extracting the texture characteristics of the wear debris. the larger the fractal dimension, the more serious the wear is.

\section{Effective analysis of metal corrosion}

The surface of the corroded metal material has fractal characteristics, which can be used to calculate the multi fractal dimension to determine the corrosion condition.

\section{Literature References}

[1] Mandelbrot. B. The fractal geometry of nature [M]. New York, W. H. Freeman, 1982.

[2] Sarkar. N, Chaudhuri. B. An efficient differential box-counting approach to compute fractal dimension of image [J]. IEEE Transactions on Systems, Man and Cybernetics, 1994, 24 (1): 115-120.

[3] Chaudhuri. B, Sarkar. N. An Efficient Approach to Compute Fractal Dimension in Texture Image[C].Proc. IEEE 11th IAPR, Conference A: Computer Vision \& Applications, 1992, 1: 358-361.

[4] N Sarkar, B.B. Chaudhuri, An efficient approach to estimate fractal dimension of textural images Pattern Recognition, Volume 25, Issue 9, September 1992, Pages 1035-1041.

[5] Kaewaramsri Y.,Woraratpanya K.Improved Triangle Box-Counting Method for Fractal Dimension Estimation, Recent Advances in Information and Communication Technology 2015 pp 53-61

[6] Shibao Lua, Jianhua Wangb, Yangang Xue, Study on multi-fractal fault diagnosis based on EMD fusion in hydraulic engineering; Applied Thermal Engineering Volume 103, 25 June 2016, Pages 798-806

[7] H.F. Huanga, X.L. Songa, C. Liua, X.Z. Huaib, A novel fractal method for fault diagnosis and signal measurements, Optik - International Journal for Light and Electron Optics,Volume 127, Issue 16, August 2016, Pages 6805-6812

[8] Hongmei Liua, 1, Jichang Zhanga, 1, Yujie Chenga, b, 1, Chen Lua, b, Fault diagnosis of gearbox using empirical mode decomposition and multi-fractal detrended cross-correlation analysis, Journal of Sound and Vibration Volume 385, 22 December 2016, Pages 350-371 